Radial and Nonradial Pulsations as Probes of Stellar Physics

ASP Conference Series, Vol. 259, 2002

C. Aerts, T.R. Bedding, $\&$ J. Christensen-Dalsgaard, eds.

\title{
A Flower on a WIRE: Asteroseismology of a Massive Star
}

\section{J. Cuypers}

Koninklijke Sterrenwacht van België, Ringlaan 3, B-1180 Brussel, Belgium

C. Aerts

Instituut voor Sterrenkunde, Katholieke Universiteit Leuven, Celestijnenlaan 200B, B-3030 Heverlee, Belgium

D. Buzasi

Department of Physics, US Air Force Academy, Colorado Springs, CO 80840, USA

\section{J. Catanzarite, T. Conrow}

Infrared Processing and Analysis Center, Mailstop 100-22, California Institute of Technology, Pasadena, CA 91125, U.S.A.

\section{R. Laher}

SIRTF Science Center, Mailstop 314-6, California Institute of Technology, Pasadena, CA 91125, U.S.A

\begin{abstract}
Recently it became clear from a line profile study that the $\beta$ Cephei star $\beta$ Crucis (Mimosa) is multiperiodic with at least three modes excited. Observations of this star by the star tracker of the WIRE satellite have now confirmed the multiperiodicity in the light variations. A total of 5 million observations covering 17 days was analysed and the three main periods are in perfect agreement with the line profile results. The amplitudes are small (3,2.7 and 0.6 millimag for the dominant modes), but this is not surprising in view of the mode identifications derived from the line profiles. More periods could still be present, including the one earlier suggested by the radial velocity data.
\end{abstract}

\section{Introduction}

Light and radial velocity variations in the $\beta$ Cephei star $\beta$ Crucis (Mimosa, HD 111123) were already observed long ago (Wright, 1909; Pagel, 1956; Van Hoof, 1962). Indications of binarity were put forward by Heintz (1957) and later confirmed by others.

Cuypers (1983) performed a period analysis of all photometric data available at that time (1959 to 1975). A main pulsation period of $0.1911846 \mathrm{~d}$, corresponding to a frequency of $5.23055 \mathrm{c} / \mathrm{d}$ (hereafter called $f_{1}$ ) was retained. No unam- 


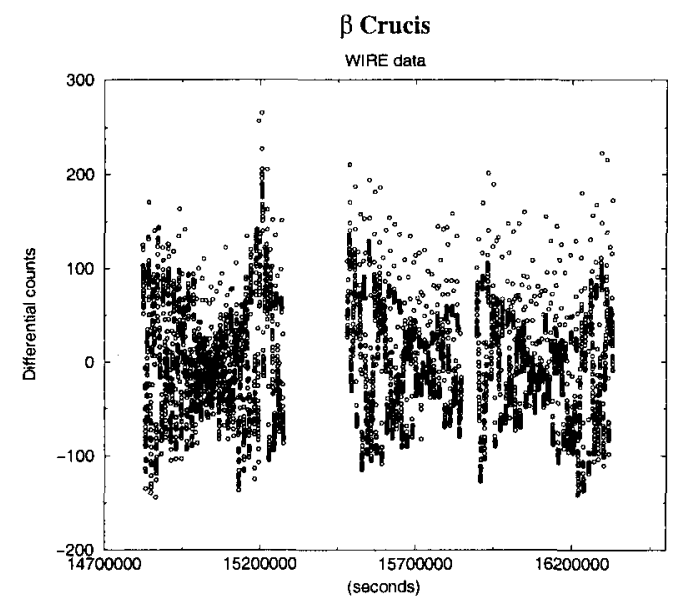

Figure 1. Raw binned WIRE data of $\beta$ Crucis.

biguous secondary period could be established. Only the frequency $2.7874 \mathrm{c} / \mathrm{d}$ was found marginally significant in both the radial velocity and the light variations.

A huge amount of spectroscopic data spanning almost 13 years was analysed by Aerts et al. (1998). The binary nature of $\beta$ Crucis was again confirmed and an unambiguous orbital period of 5 years was deduced. Multiperiodicity was clearly visible in the line profile variations. Two more frequencies $\left(f_{2}=5.958666 \mathrm{c} / \mathrm{d}\right.$ and $f_{3}=5.472165 \mathrm{c} / \mathrm{d}$ ) were detected, in addition to the one already found present in the light variations. More variability was suspected. Aerts et al. (1998) attempted identification of the pulsation modes, and found that none of the modes is radial, and that the newly detected frequencies have all $\ell \geq 3$.

$\beta$ Crucis was observed intensively by the star tracker of the WIRE satellite. Buzasi et al. (2000), Buzasi (these proceedings) and Cuypers et al. (2002) described how this star tracker could be used for asteroseismology and other variable star observations. Here we present preliminary results from the $\beta$ Crucis data and we comment on the amplitudes in view of the mode identifications obtained earlier.

\section{WIRE results}

$\beta$ Crucis was observed by the WIRE satellite during 17 days, with a large gap of about 2.4 days and a shorter second one. Near the end of the first period there was a sudden brightening of $0.01 \mathrm{mag}$ of the star. This is probably instrumental, but the cause is still unknown. These data were disregarded. At a rate of $10 \mathrm{~Hz}$ about 5 million measurements of the star were collected. These data came in blocks of about 38 minutes per 96 -minute orbit.

A lot of features correlated with the orbital period were seen and correcting for these was not easy a priori. However, a first look at the data immediately 


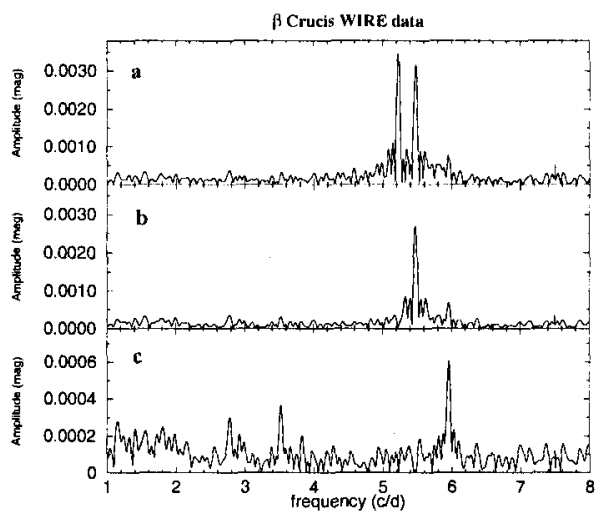

Figure 2. Lomb-Scargle periodograms of the WIRE data of $\beta$ Cru: original data (a), after prewhitening for $f_{1}(\mathrm{~b})$ and after prewhitening for $f_{1}$ and $f_{2}(\mathrm{c})$. In (c) the scale of the $\mathrm{y}$ axis has been changed.

reveals a clear beat pattern: at least two frequencies are present (Fig. 1). Several binning schemes were used, but since the expected light variation periods were considerably longer ( 4 hours) than the satellites orbital period, we decided to use only the mean value of each orbit with observations. This resulted in 214 very high quality data points.

Frequency analysis of the 214 points (Fig. 2) immediately led to two frequencies: 5.228 and $5.477 \mathrm{c} / \mathrm{d}$. They are extremely close to the frequencies $f_{1}$ and $f_{3}$ of the line profile variations. A third frequency could also be identified without ambiguity: $5.956 \mathrm{c} / \mathrm{d}$. This is, within the errors, equal to the frequency $f_{2}$ of the spectroscopic analysis.

After prewhitening there is still variability present in the residuals. The amplitudes of the frequencies $f_{4}=3.527 \mathrm{c} / \mathrm{d}$ and $f_{5}=2.785 \mathrm{c} / \mathrm{d}$ are not high above the noise level, estimated by us to be smaller than $0.0002 \mathrm{mag}$, but $f_{5}$ coincides precisely with the secondary frequency suggested earlier by Cuypers (1983). The 3 main frequencies are also present in the second, 9-day, data block. In the first data block, $f_{1}$ and $f_{3}$ could not be separated, since its length was only 5 days. The same results were also obtained from the non-binned data.

\section{Amplitudes and mode identifications}

Although no multi-colour photometry over a long time interval is available, we can check whether the amplitudes found are in agreement with the previous spectroscopic mode identifications $\left(\ell=1\right.$ for $f_{1}, \ell=3$ for $f_{2}, \ell=4$ for $\left.f_{3}\right)$. We used therefore the relative amplitude estimates as a function of wavelength and mode as described by Heynderickx et al. (1994) with the parameters for $\beta$ Crucis as given in Aerts et al. (1998).

The spectral response of the WIRE star tracker is approximately equivalent to a $V+R$ filter, while the photometric data by Van Hoof (1962) and re-analysed 
by Cuypers (1983) were obtained with a $U$ filter. For all modes a decrease of the amplitudes towards the red is expected. The amplitude of $f_{1}$ as observed by WIRE $(0.0031 \mathrm{mag})$ is smaller than the expected $70 \%$ of the $\mathrm{U}$ amplitude of $0.012 \mathrm{mag}$ for mode $\ell=1$, but no other $\ell$ value gives a better result. It is no surprise that $f_{2}$ with an amplitude of only $0.0006 \mathrm{mag}$ was never identified from the ground, since an $\ell=3$ mode will have at most a 0.001 mag amplitude in the visible or in U. However, the decrease in amplitude towards the red is not very large for an $\ell=4$ mode. Since the WIRE amplitude of $f_{3}$ is only $0.0027 \mathrm{mag}$, the corresponding predicted $\mathrm{U}$ amplitude is no higher than about $0.004 \mathrm{mag}$, and therefore $f_{3}$ was not previously identified in U. However, a similar amplitude should be present in the visible region. Examination of the Hipparcos data for $\beta$ Crucis (ESA, 1997) shows that a clear secondary, but only marginally significant, frequency is present at $5.4774 \mathrm{c} / \mathrm{d}$. This is extremely close to frequency $f_{3}$ and the amplitude $(0.004 \mathrm{mag})$ is in perfect agreement with our $\ell=4$ mode identification.

\section{Conclusions}

The preliminary results of the asteroseismological program with the star tracker of the WIRE satellite for the massive main sequence pulsator and supernova progenitor $\beta$ Crucis are extremely promising. The multiperiodicity previously known only from spectroscopic analysis could be confirmed in the light variations. The amplitudes of the light variations as observed by WIRE are compatible with the mode identifications as derived from the spectroscopic data.

Acknowledgments. We are very grateful to all those who made this use of the WIRE satellite possible. JC acknowledges support from the Belgian Fund for Scientific Research - Flanders (G.0265.97).

\section{References}

Aerts C., De Cat P., Cuypers J., Becker S.R., Mathias P., Gillet D., \& Waelkens C. 1998, A\&A, 329, 137

Buzasi, D., Catanzarite, J., Laher, R., Conrow, T., Shupe, D., Gautier III, T.N., Kreidl, T., \& Everett, D. 2000, ApJ 532, L133

Cuypers, J. 1983, A\&A, 127, 186

Cuypers, J., Aerts, C., Buzasi, D., Catanzarite, J., Conrow, T., \& Laher, R., 2002 , in The 1st Eddington Workshop, ESA SP-485, in press

ESA, 1997, The Hipparcos and Tycho Catalogues, ESA SP-1200

Heintz, W.D. 1957, Observatory 77, 200

Heynderickx, D., Waelkens, C., \& Smeyers, P., 1994, A\&AS, 105, 447

Pagel, B.E.J. 1956, MNRAS 116, 10

Van Hoof, A. 1962, Z. Astrophys., 54, 244

Wright, W.H. 1909, Lick Obs. Bull. 5, 177 